\title{
Erratum notice for: "SAD-B modulates epileptic seizure by regulating AMPA receptors in patients with temporal lobe epilepsy and in the PTZ-induced epileptic model" [Braz J Med Biol Res (2020) 53(4): e9175]
}

\begin{abstract}
Rong Li ${ }^{1}$, Miaoqing He(i) ${ }^{2,3}$, Bing Wu(i) ${ }^{4}$, Peng Zhang ${ }^{1}{ }^{1}$, Qinbin Zhang ${ }^{1}{ }^{1}$, and Yangmei Chen (i) ${ }^{1}$
${ }^{1}$ Department of Neurology, Second Affiliated Hospital of Chongqing Medical University, Chongqing, China ${ }^{2}$ Center for Brain Disorders Research, Capital Medical University, Feng Tai District, Beijing, China ${ }^{3}$ Beijing Institute for Brain Disorders, Feng Tai District, Beijing, China ${ }^{4}$ Department of Neurology, First Affiliated Hospital of Chongqing Medical University, Chongqing Key Laboratory of Neurology, Chongqing, China
\end{abstract}

Erratum for: Braz J Med Biol Res | doi: 10.1590/1414-431X20199175 | PMID: 32267308 | PMCID: PMC7162585

On July 7, 2020, the Brazilian Journal of Medical and Biological Research received a request from the first author Rong $\mathrm{Li}$ requesting the substitution of Figure 1B, Panels: Rat Hippocampus SAD-B, MAP2, and MERGE because these 3 images had been submitted incorrectly. This modification of Figure 1 does not change the findings of this research. After careful evaluation by the Editors, this erratum is being published.

A
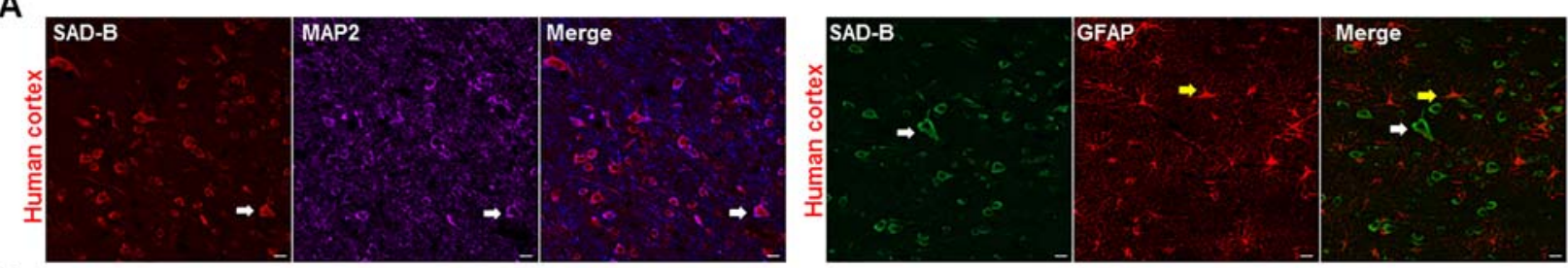

B
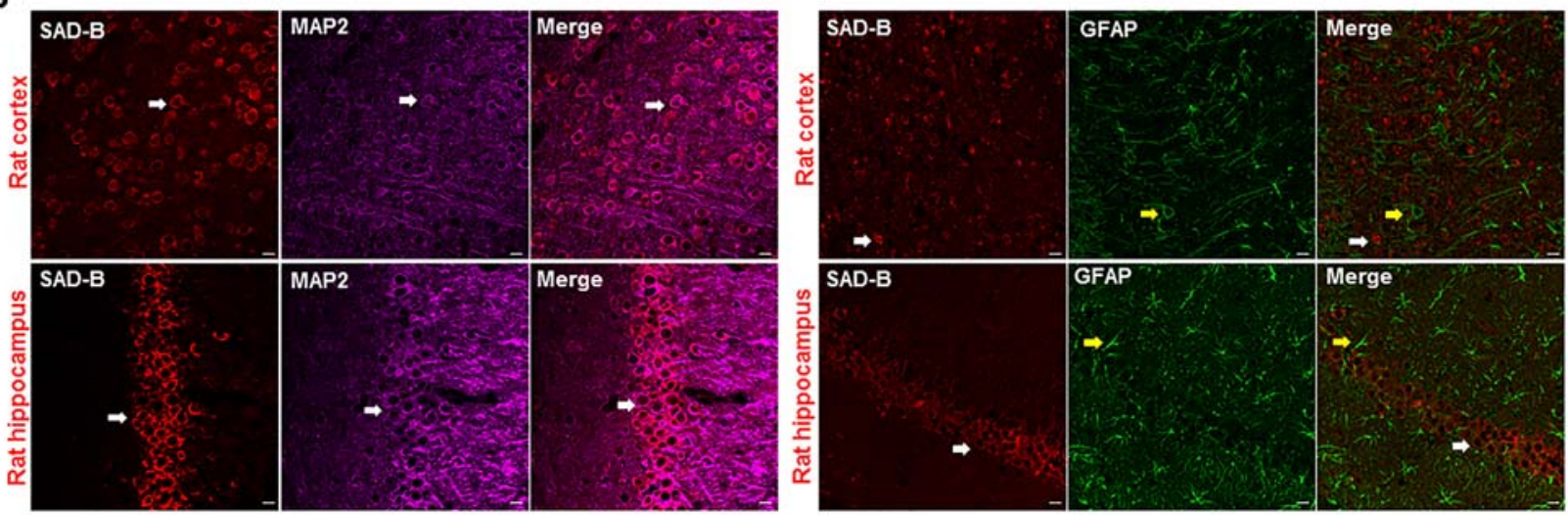

Figure 1. Brain-specific serine/threonine-protein kinase 1 (SAD-B) is localized in the epileptic brain. A, Immunofluorescence labelling of SAD-B (red), MAP2 (violet), and GFAP (green) in the cortex of patients with temporal lobe epilepsy (TLE) showing that SAD-B was co-localized with MAP2 but not with GFAP. Scale bar: $50 \mu \mathrm{m}(400 \times)$. B, Immunofluorescence labelling of SAD-B (red), MAP2 (violet), and GFAP (green) in the CA1 region of the hippocampus or cortex of an epileptic rat showing that SAD-B was co-localized with MAP2, but not with GFAP. Scale bar: $50 \mu \mathrm{m}(400 \times)$. White arrows: SAD-B; yellow arrows: GFAP. 